\title{
Women and Heart Disease; A Focus Issue
}

\author{
Jawahar L. Mehta ${ }^{1}$
}

Published online: 31 July 2015

(C) Springer Science+Business Media New York 2015

While coronary heart disease (CHD) as a major cause of death among men has been known for almost half a century, it is only in the last 2 decades that CHD is widely recognized as the most common cause of death among women.

Clinical studies funded in a large part by the United States National Institutes of Health have revealed major differences in the pathogenesis and presentation of CHD in men and women. It has also become clear that there are real differences in the outcome of CHD in men and women. Some of the differences may relate to hormonal differences in men and women, primarily estrogen levels, which decline rapidly during the menopause.

Although estrogen was thought to be the basis of lower prevalence of CHD among women almost since its discovery, it was in mid-1960s when Dr. Robert Watson published the book Feminine Forever and started to promote premarin as an elixir of life that would maintain women's youthfulness and prevent many of the diseases affecting men like CHD and cancers. Premarin and other estrogen preparations were prescribed in large quantities to keep away the hot flashes and other unpleasant symptoms of menopause. In the 1980s, there were reports of increased risk of cancer of the uterus with estrogen intake. In 2001, the Women's Health Initiative study showed that estrogens not only did not prevent CHD but actually increased it.

Work done by a number of prominent investigators defined not only the role of sex hormones in CHD, but also the role of

Jawahar L. Mehta

mehtajl@uams.edu

1 Medicine and Physiology and Biophysics, Stebbins Chair in Cardiology, University of Arkansas for Medical Sciences, Little Rock, AR 72205, USA metabolic syndrome (dyslipidemia, hypertension, altered insulin sensitivity and increase in body weight) in the development of atherosclerosis.

At an editorial board meeting of Cardiovascular Drugs and Therapy, Prof. Remme, the Editor-in-Chief, and I thought of putting together a focus issue of the Journal relating to CHD in women and to highlight the differences in men and women with regard to the pathogenesis of atherosclerosis and hypertension, regulation of myocardial blood flow, presentation of this malady, and differences in the treatment, especially the use of pharmacotherapy.

Although not a complete encyclopedia on the vast topic of CHD, this issue presents select aspects of CHD in women.

Mathur et al. [1] very succinctly but completely discuss the data on various aspects of gender-related differences in atherogenesis with emphasis on the effects of sex hormones, differences in plaque morphology, and vascular endothelial function.

Rochlani et al. [2] discuss various aspects of metabolic syndrome-epidemiology of this syndrome in many parts of the world, significant sex disparities in metabolic syndrome components, and genetic and hormonal influences that could impact the development of CHD in women.

Cenko and Bugiardini [3], based on their vast experience, write that a large proportion of women have normal coronary arteries on angiography without any significant evidence of flow-limiting disease but have biochemical and/or imaging evidence of myocardial ischemia. In these women, they suggest that it may well be a dysfunction of coronary microcirculation and/or macrocirculation, or vasotonic angina that leads to abnormal vasoconstriction, and potentially to myocardial infarction, ventricular arrhythmias, and sudden death.

Epidemiologic studies show differences in left ventricular mass in hypertensive men and women, and left ventricular mass is an independent index of mortality. Wittnich et al. [4] 
discuss the role of $17 \beta$-estradiol in hypertension and the cardiovascular system. They show based on animal studies that plasma estradiol modulates relative heart mass in both normotensive and hypertensive female rats. They show that hypertensive females exhibit a blunted response to $17 \beta$-estradiol compared with the normotensive females with an increase in plasma $17 \beta$-estradiol, which may be related to a reduced estrogen receptor expression in the presence of hypertension.

Brewer and colleagues [5] review the current challenges of prevention, diagnosis and treatment of ischemic heart disease in women. They discuss that women often present with atypical symptoms, and this often leads to underdiagnosis or late diagnosis, and hence undertreatment of CHD in women. They also suggest that women suffer more disability and poorer clinical outcomes, with higher cardiovascular morbidity and mortality, than men. Like Cenko and Bugiardini [3], they emphasize that the unusual presentation of CHD in women may in part be secondary to the higher prevalence of nonobstructive coronary artery disease with persistent chest pain symptoms when evaluated invasively.

Bhatt et al. [6] discuss the differences in men and women in terms of CHD in India, which represents a fast developing market with a rapid change in women's role in society. They show that in India women appear to have a higher prevalence of hypertension, diabetes and obesity. Importantly, women get invasive treatments less often than men. Depression is also more common in women than in men and is associated with a poor quality of life and higher mortality.

Kenchaiah and Vasan [7] report insights into sex-based differences in the epidemiology of heart failure based primarily on the Framingham Heart Study database. They report that among those without prior myocardial infarction, the lifetime risk of heart failure is approximately $15 \%$ for women and $11 \%$ for men, and hypertension is associated with a significantly higher risk of heart failure. Similarly, heart failure occurs almost twice as often in women with diabetes than in men.

Park et al. [8] discuss a novel topic of adverse pregnancy conditions, infertility, and future cardiovascular risk. They suggest that adverse pregnancy outcomes such as preterm delivery and low birth weight may manifest as a risk factor for cardiovascular disease later in maternal life.
Stolarz and Rusch [9] present evidence for gender- related differences in the responses to common cardiovascular drugs, including statins, antiplatelet and antithrombotic agents, $\beta$ blockers, digoxin and drugs associated with the long QT syndrome. They point out that the QT interval of the electrocardiogram is longer in women compared to men, and as such drugs that prolong the QT interval are more likely to cause lethal ventricular arrhythmias in female than in male patients.

It is my hope that this focus issue will stimulate further research in the important area of heart disease in women, and define the gender-based differences in the pathogenesis of atherosclerosis, and lead to new techniques that will improve clinical diagnosis and improved therapy of CHD and related maladies in women.

I want to thank Prof. Remme for giving me the opportunity to edit this focus issue, his staff, particularly Mrs. Noor van Dam, and Ms. Lisa Aquilino of Springer Publishing for making this issue possible.

\section{References}

1. Mathur P, Ostadol B, Romeo F, Mehta JL. Gender-related differences in atherosclerosis. Cardiovasc Drugs Therapy 2015;29: this issue

2. Rochlani Y, Pothineni NV, Mehta JL. Metabolic syndrome: does it differ between women and men? Cardiovasc Drugs Therapy 2015;29: this issue

3. Cenko E, Bugiardini R. Vasotonic angina as a cause of myocardial ischemia in women. Cardiovasc Drugs Therapy 2015;29: this issue

4. Wittnich $\mathrm{C}$, Wallen J, Belanger M. The role of $17 \beta$-estradiol in myocardial hypertrophy in females in the presence and absence of hypertension. Cardiovasc Drugs Therapy 2015;29: this issue

5. Brewer LC, Svatikova A, Mulvagh S. The challenges of prevention, diagnosis and treatment of ischemic heart disease in women. Cardiovasc Drugs Therapy 2015;29: this issue

6. Bhatt P, Parikh P, Patel A, Parikh R, Patel A, Mehta JL, Parikh K. Unique aspects of coronary artery disease in Indian women. Cardiovasc Drugs Therapy 2015;29: this issue

7. Kenchaiah S, Vasan RS, Heart failure in women - insights from the Framingham Heart Study. Cardiovasc Drugs Therapy 2015;29: this issue

8. Park K, Wei J, Minissian M, Bairey CN, Pepine CJ. Adverse pregnancy conditions, infertility, and future cardiovascular risk: implications for mother and child. Cardiovasc Drugs Therapy 2015;29: this issue

9. Stolarz AJ, Rusch NJ. Gender differences in the response to cardiovascular drugs. Cardiovasc Drugs Therapy 2015;29: this issue 